\title{
A survey of human histone H1 subtypes interaction networks: implications for histones H1 functioning
}

\author{
Andrzej Kowalski ${ }^{1}$ \\ ${ }^{1}$ Jan Kochanowski University in Kielce
}

September 24, 2020

\begin{abstract}
Human histone H1 subtypes interaction networks was constructed to show a spectrum of their activities realized through the protein-protein interactions. Histone H1 subtypes participate in over half a thousand interactions with nuclear and cytosolic proteins engaged in the enzymatic activity and binding of nucleic acids and proteins. Small scale networks created by H1 subtypes are similar in their topological parameters $(\mathrm{p}>0.05)$ but hub proteins of the networks formed with subtype H1.1 and H1.4 differ from those of subtype H1.3 and H1.5 in the closeness centrality, clustering coefficient and neighborhood connectivity $(\mathrm{p}<0.05)$. Molecular function and biological process of the networks hubs is related to RNA binding and ribosome biogenesis (subtype H1.1 and H1.4), cell cycle and cell division (subtype H1.3 and H1.5) and protein ubiquitination and degradation (subtype H1.2). Such a disparity between H1 subtypes is also manifested by enriched GO terms of their interacting proteins. The residue propensity and secondary structures of interacting surfaces as well as a value of equilibrium dissociation constant indicate that a type of $\mathrm{H} 1$ subtypes interactions is transient in term of the stability and medium-strong in relation to the strength of binding. Histone H1 subtypes bind interacting partners in the intrinsic disorder-dependent mode, according to the coupled folding and binding and mutual synergistic folding mechanism. These results evidence that multifunctional H1 subtypes operate via protein interactions in the networks of crucial cellular processes and, therefore, confirm a new histone H1 paradigm relating to its functioning in the protein-protein interaction networks.
\end{abstract}

\section{1 | INTRODUCTION}

Usually, protein exert its biological function forming a pair with other protein as a result of interaction. Due to a great diversity of variously created protein complexes, the protein-protein interactions might be defined in several contexts. They are direct when proteins stay in close, physical, contact with other protein or indirect when interaction between proteins is maintained by intermediate in the complex. ${ }^{1} \mathrm{In}$ respect of a stability, protein-protein interaction might be stable and irreversible, i.e. permanent, or able to temporal association/dissociation, i.e. transient. In terms of binding affinity, the permanent interactions are usually obligate, i.e. including components that cannot exist independently, while transient are non-obligate, i.e. including constituents able to exist independently. ${ }^{2}$ Irrespective of the constituents of interactions and a type of created complexes, the interactions between proteins are crucial for a right course of all cellular processes running in the various cellular structures. ${ }^{3}$ One of them is chromatin, a multi-subunit complex contained over 1900 proteins participating in DNA templated processes, such as gene regulation, packaging and repair. ${ }^{4}$ In chromatin, a highly abundant are histones. They represent five classes of proteins, i.e. H2A, $\mathrm{H} 2 \mathrm{~B}, \mathrm{H} 3, \mathrm{H} 4$ and $\mathrm{H} 1$, responsible for organization of chromatin structure and modulation of its genetic and epigenetic functioning. ${ }^{5}$

Histone H1 is well-known as core component of chromatin implicated in maintenance of nucleosomal and linker DNA to promote chromatin compaction and formation of its higher order structure. ${ }^{6,7}$ Such canonical account of histone $\mathrm{H} 1$ functioning is nowadays supplemented by its activity realized through the interactions with proteins that differ in their cellular localization and belongs to distinct functional categories. They 
include spliceosome-associated proteins, core histone binding chaperone proteins, regulatory transcription proteins, DNA damage repair proteins and translation-associated proteins. ${ }^{8}$ Thus, histone H1 extended paradigm point that it should be seen as multifunctional protein engaged in the course of diverse biological processes. However, a vast majority of known histone $\mathrm{H} 1$ partnering proteins refers to the one human histone H1 subtype, i.e. H1.0. To know a broader range of histone $\mathrm{H} 1$ activities realized via interaction with partner proteins, a successive analyzes concerning subsequent histone H1 subtypes are needed. This work is aimed at fill this gap through the characteristic of proteins that interact with five standard human histone H1 somatic subtypes (H1.1 - H1.5). To do this, the identification of histone H1 partnering proteins subcellular localization was performed together with prediction of their molecular function and biological processes. Besides, the histone H1 interaction networks were constructed to show a scope of their activity. Based on the secondary structure motifs and amino acid composition of proteins binding interfaces, a strength of protein-protein interaction and a type of formed complexes was appointed. Finally, an impact of intrinsic structural disorder on protein-protein interaction was determined to define a mechanism of protein complex formation. A scheme depicting the data processing stages is presented in the Fig.1.

\section{2 | METHODS}

\subsection{Identification and processing of histone H1 subtypes interacting proteins}

The partnering proteins of five standard human histone H1 somatic subtypes (H1.1 - H1.5) were found in the APID (Agile Proteins Interactomes DataServer) database. ${ }^{9}$ APID contain the records of proteinprotein interactions supported by detection methods evidencing a direct physical interaction between the protein pair, i.e. binary interactions, and identified by co-complex associations, i.e. indirect interactions. A type of binary interaction was derived from the records of BioGRID (Biological General Repository for Interaction Datasets) ${ }^{10}$ and IntAct (Molecular Interaction Database) ${ }^{11}$ databases, a literature curated sources of molecular interaction data. The sequences of selected proteins were searched in the resource of protein sequence and annotation data UniProt ${ }^{12}$ and further analyzed in the FASTA format.

\section{2 | Determination of histone H1 subtypes interacting proteins localization}

Localization of histone H1 subtypes partnering proteins was determined by searching the ComPPI (Compartmentalized Protein-Protein Interaction Database) database, the integrated source constructed based on the eight localization databases. ComPPI evidence protein localization by experimentally verified methods predicted by computational and unknown type data with localization score, i.e. the probability of a given protein to be found in a certain compartment, weighted as $\mathrm{p}=0.8, \mathrm{p}=0.7$ and $\mathrm{p}=0.3$, respectively. ${ }^{13}$

\section{3 | Determination of histone H1 subtypes interacting proteins function}

Functions assigned to the partnering proteins of histone H1 subtypes were provided by SIFTER (Statistical Interference of Function Through Evolutionary Relationships). This is a statistical approach for prediction protein function with the use of a protein family's phylogenetic tree as the natural structure for representing protein relationships. Protein function is defined with the confidence score, a value between 0 and 1 , calculated based on the number of candidate functions and family size as well as the frequency and distribution of candidate terms in the tree. ${ }^{14}$

\section{4 | Determination of histone H1 subtypes interacting proteins GO terms}

Protein characterization with respect to the GO annotations, i.e. biological process, cellular component and molecular function, was done with CELLO2GO (subCELlular LOcalization Prediction with Functional Gene Ontology Annotation) and a g:GOSt tool of g:Profiler. The CELLO2GO is a tool that use BLAST for search a similarity of protein query sequence to the sequences of proteins which GO categories are deposited in the UniProt database. ${ }^{15}$ The g:GOSt perform functional analysis identifying a statistically significantly enriched GO annotations using g:SCS (Set Counts and Sizes) correction method. ${ }^{16}$

\section{5 | Generation of histone H1 subtypes and interacting proteins networks}


Histone H1-partner proteins interaction networks were created with STRING (Search Tool for the Retrieval of Interacting Genes/Proteins) platform (Version 11.0). ${ }^{17}$ Protein-protein interaction data were predicted based on the active interaction sources including seven available parameters, i.e. co-expression, co-occurrence, databases, experiments, gene fusion, neighborhood and text mining, with medium (0.400) confidence interaction score. A STRING graph networks were further analyzed with Cytoscape (version 3.8.0) plugin app Network Analyzer ${ }^{18}$ based on several topological parameters such as average number of neighbors (the average connectivity of a node in the network), network diameter (the largest distance between two nodes), network radius (the minimum among the non-zero eccentricites of the nodes in the network), network heterogeneity (the tendency of a network to contain hub nodes), network clustering coefficient (the average of the clustering coefficients for all nodes in the network) and the number of connected components (the connectivity of a network). An identification of hub objects in the networks was performed with the use of cytoHubba Cytoscape plugin, adopting the maximal clique centrality method (MCM) which was proposed as most effective in the exploration of the hub nodes in the network. ${ }^{19}$ To find subnetworks, a Diffusion tool which is an integrated Cytoscape function for performing a network propagation ${ }^{20}$ was adopted.

2.6 Prediction of histone H1 subtypes and interacting proteins residues contact and binding sites

Binding sites in the protein sequences were predicted with BIPSPI (xgBoost based Interface Prediction of Specific Partner Interactions), a partner-specific predictor of residue-residue contacts and protein binding sites. BIPSPI is a machine learning-based method employs Extreme Gradient Boosting (XGBoost) models trained on the input sequences pairs of the protein complexes. ${ }^{21}$

\section{7 | Prediction of disorder in the histone H1 subtypes and interacting proteins}

Disorder in the histone H1 subtypes and partnering proteins sequences was determined with DisEMBL Hot loops definition, assumes that a highly dynamic loops determined from $\mathrm{C}_{\alpha}$ temperature factors varying greatly in a single protein structure corresponds to the protein disorder. ${ }^{22}$

2.8 - Prediction of secondary structure types in the histone $\mathrm{H} 1$ subtypes and interacting proteins

Secondary structure prediction was done by GOR (Garnier-Osguthorpe-Robson) V method. It combine an original GOR algorithm, using the information theory and Bayesian statistics, with evolutionary information originating from PSI-BLAST multiple sequence alignments. ${ }^{23}$

\section{9 - Prediction of binding affinity between histone H1 subtypes and interacting proteins}

Binding affinity of histone $\mathrm{H} 1$ subtypes and their partner proteins was determined with ISLAND (In-Silico Prediction of Proteins Binding Affinity Using Sequence Descriptors), a sequence-based only predictor trained on the regression models (OLSR, RFR and SVR) along with various types of sequence descriptors with LOCO cross-validation over the docking benchmark dataset. ${ }^{24}$

\subsection{0 - Statistical analysis}

The one-way ANOVA test was applied for evaluation a difference in the localization, function and the network topological parameters between histone H1 subtypes partnering proteins. The strength of relationship between the network parameters was measured using Pearson correlation coefficient. The Mann-Whitney $\mathrm{U}$ test was employed for comparison the parameters of top hub proteins in the histone H1 subtype-protein interacting networks. In all analysis, a p value $>0.05$ was regarded as statistically significant for rejecting the null hypothesis.

\section{3 - RESULTS AND DISCUSSIONS}

\section{1 - Identification of histone $\mathrm{H1}$ subtypes interacting proteins}

The partner proteins of standard histone H1 somatic subtypes, i.e. H1.1 - H1.5, were retrieved from the APID analyzer by enter the UniProt accession number (AC) of a given histone H1 subtype. According to 
the decreasing amount of interacting proteins, the histone H1 subtypes takes the following order: H1.1 (190), H1.4 (133), H1.2 (123), H1.5 (71) and H1.3 (47) (Table S1 - S5). The vast majority of interactions, i.e. 93.6\%, were indicated as indirect, meaning that proteins have only functional relationship without direct physical contact. Due to that $4.3 \%$ of interactions were not annotated, the binary interactions represent $2.1 \%$ only. They related to the pair of proteins that stay in the close contact and able to form a complex. From all of 564 proteins identified as interacting with histone $\mathrm{H} 1$ subtypes, 343 proteins are the partners for a given histone H1 subtype only. They represent more than half of all proteins interacting with histone H1.1 (68.9\%), H1.2 (60.2\%), H1.4 (62.4\%) and H1.5 (50.7\%) and almost half that interact with histone H1.3 (40.4\%) (Figure 2A). The remained proteins are the partners characteristic of more than one H1 subtype. Usually for two subtypes, alike protein-lysine 6-oxidase interacting with the histone H1.1 and H1.5 and suppressor of SWI4 1 homolog identified as a partner of subtype H1.2 and H1.4. A less frequently represented are proteins which interact with three and four histone H1 subtypes. They include, among others, the DNA replication licensing factor MCM5 which is a partner of subtype H1.1, H1.2 and H1.4. Likewise, a 60S ribosomal protein L28 is involved in the interaction with subtype H1.1, H1.2 and H1.3 and a chromobox protein homolog 6 is a partner of subtype H1.1, H1.2, H1.4 and H1.5. A common partners for all histone H1 subtypes are nucleophosmin, interleukin-1 receptor-associated kinase 4 and protein kinase $\mathrm{C}$ alpha type. A pairwise grouping of histones $\mathrm{H} 1$ in relation to the common interacting proteins raises supposition that subtypes sharing more same partners possesses a similar activity. The subtypes H1.1 and H1.4 having about 30 same interacting proteins may display similar functional properties. In contrast, subtypes H1.3 and H1.4 possessing up to 5 same proteins can be considered as more functionally distinct (Figure 2B). The observed quantitative disproportions between common and individual partnering proteins indicate that histone H1 subtypes activity may be both specific and general, corresponding to the partial redundancy. ${ }^{25}$

\section{2 - Localization of histone H1 subtypes interacting proteins}

Localization of histone H1 subtypes partnering proteins was determined with highest localization score indicated in the ComPPI database. As it was shown in the Figure 3, the main subcellular compartment in which the proteins are located is nucleus and cytosol, characteristic of $46.0-65.0 \%$ and $22.0-40.0 \%$ proteins, respectively. Although no statistically significant difference in the localization of histone H1 subtypes partnering proteins was detected $(F=0.0052, \mathrm{p}=0.999)$, a disproportions of their emplacement shows on a diverse engagement of histone $\mathrm{H} 1$ subtypes in the processes running in the nucleus and cytosol. Whereas histone H1.1 have $65.0 \%$ of partner proteins located in the nucleus and $22.0 \%$ characteristic of a cytosol, it should be perceived as more active in the nucleus. In contrast, the histone H1.2, possessing $48.0 \%$ partnering proteins in the nucleus and $40.0 \%$ in the cytosol, might be similarly active in both regions of the cell. Likewise, almost twice more histone H1.4 interacting proteins originating from nucleus $(59.0 \%)$ than from cytosol (30\%) indicate that this histone H1 subtype may play more significant role in the nucleus. On the contrary, a participation of histone H1.3 in the processes running in the nucleus (46.0\% partnering proteins) and in the cytosol (36.0\% partnering proteins) can be considered as comparable. Other proteins interacting with histone $\mathrm{H} 1$ subtypes are the components of membrane, mitochondrion and secretory pathway and also remain in both nucleus and cytosol. Such a locations are generally characteristic of less than $5 \%$ of proteins, except for these which are the partners of histone H1.1 located in both nucleus and cytosol (6.0\%) as well as the membrane proteins (10.0\%) interacting with histone H1.3. Besides, a comparable proportions of the secretory pathway proteins designated as the partners of histone H1.1 - H1.4 (1.0 - 2.0\%) differ from an amount of proteins predicted as interacting with histone H1.5 (9\%). This suggest a stronger relationship of histone H1.5 with function of the endoplasmic reticulum and Golgi apparatus. Although a complete wide-genome distribution of $\mathrm{H} 1$ subtypes still needs to be definitively determined, a known reports regarding localization of $\mathrm{H} 1$ histones point to their association with a specific chromatin type and, thus, a characteristic role in chromatin-dependent cellular processes. Based on the findings of Izzo et al. ${ }^{26}$, a disparate abundance of histone H1.1 and histone H1.2 - H1.5 are discernible at promoters, enhancers and CpG islands. Histone H1.1 is preferentially located in the PcG regions marked by H3K9me3 while histone H1.2 - H1.5 in the HP1-containing domains marked by H3K27me3. Thus, in contrast to the subtype H1.2 - H1.5, subtype H1.1 has more potential to form both active and repressed chromatin state. In another study ${ }^{27}$, a difference in 
emplacement in the genome was detected between histone H1.2 and other H1 subtypes. Whereas histone H1.0 and H1X was detected as associated with high CG content and gene-rich regions, histone H1.2 was indicated as located in the regions with low CG content and gene-poor. These reports pointing to peculiar distribution of histone $\mathrm{H} 1$ subtypes reflects simultaneously their differential influence on processes running in the chromatin. It seems that a similar relationship may concern the activity of histone H1 subtypes differently realized in various cell structures through their interaction with partner proteins.

\section{3 - Functions of histone H1 subtypes interacting proteins}

The four main functional categories of H1 partner proteins indicated in the SIFTER database include enzymatic activity and binding of the proteins, DNA and RNA (Figure 4). Although proportions of proteins assigned to a given functional category were detected as statistically insignificant between histone H1 subtypes $(\mathrm{F}=0, \mathrm{p}=1)$, a distribution of proteins representing various activities is non-uniform. This suggest that H1 subtypes are differently engaged in the protein and nucleic acids-dependent events. Histone H1.2, H1.3 and H1.5, having more than 50\% interacting proteins responsible of protein binding, might more frequently realize its function via protein-protein interaction than through the interaction with nucleic acids, due to that their partner proteins are able to bind them in at most $10 \%$. A binding of proteins is attributed to the chromatin builders, e.g. histone H2A-Bbd type $2 / 3$ and histone H3.3, and regulators, e.g. PHD finger protein 6 and protein-lysine 6 -oxidase, but also to the factors involved in the protein degradation by ubiquitination, e.g. cullin $4 \mathrm{~B}$ and cullin 5 . The other proteins are engaged in cell adhesion and signaling, e.g. intercellular adhesion molecule 1 and integrin alpha-4, as well as in the control of cell cycle, e.g. G1/S-specific cyclin-E1 and G2/mitotic-specific cyclin-B1. Whereas proteins which bind to the DNA are poorly represented among the partners of H1 histones, in an amount not exceed 10\%, the RNA binding proteins are more abundant. Histones H1.1 and H1.4 are an examples of $\mathrm{H} 1$ subtypes displaying $21 \%$ and $34 \%$ interactions, respectively, with RNA binding partners. They include proteins that bind to the mRNA, e.g. UAP56-interacting factor, tRNA, e.g. lupus LA protein, and rRNA, eg. RNA-binding motif protein 19, thus, they might be directly involved in the biogenesis of the RNA. Moreover a group of these RNA-binding proteins, alike 60 ribosomal protein L37 and cellular nucleic acid-binding protein, are the components of translation machinery. Also, among the partners of $\mathrm{H} 1$ histones are proteins displaying the enzymatic activity, mainly such that modify histones, e.g. N-methyltransferase, H3 lysine-79 specific and histone acetyltransferase KAT2B, as well as the DNA, e.g. DNA (cytosine-5)-methyltransferase 3B, and RNA, e.g. ribosomal RNA-processing protein 8. Among the miscellaneous functions assigned to histone H1 interacting proteins, mostly represented is protein homodimerization activity, characteristic for the partners of all H1 subtypes. Likewise a zinc ion binding is represented by proteins interacting with four H1 subtypes. Besides functions assigned to the proteins interacting with several H1 histones, there are also functions characteristic of a given subtype only. For example, chromatin binding is attributable for proteins partnering subtype H1.3 and transmembrane signaling receptor for the partners of subtype H1.5. However, a highest number of partners having various functions possess subtype H1.2. Among them are nucleosome binding, protein heterodimerization activity and magnesium ion binding that are not represented by partners of other H1 subtypes. In this context, histone H1.2 can be perceived as peculiar subtype, participating in the processes specific only for them. A selective function that regard histone H1.2 is related, for instance, to its ability of gene silencing by forming compacted chromatin structure together with repressive transcription complex PRC2. ${ }^{28}$

Functional characterization of analyzed proteins as per the GO terms was done with CELLO2GO (Table 1). Among the molecular function corresponding to the proteins interacting with all of $\mathrm{H} 1$ subtypes is ion binding (GO:0043167), DNA binding (GO:0003677), RNA binding (GO:0003723) and kinase activity (GO:0016301). A rest of top molecular functions was assigned to the proteins that are partners for particular H1 subtypes only. For partner proteins of four (H1.1, H1.3, H1.4 and H1.5) and three (H1.1, H1.2 and H1.4) histone H1 subtypes, a hydrolase activity (GO:0016787) and structural constituent of ribosome (GO:0003735), respectively, is attributed. A transferase activity (GO:0016740) is characteristic for proteins interacting with subtype H1.3 and H1.5 while protein binding (GO:0005515) correspond to the partners of subtype H1.2. However, the differences in the abundance of proteins with a given GO term that interact with particular H1 subtype may suggest its weaker and/or stronger preferences to fulfill a specific function. 
Thus, in contrast to other H1 histones, the subtype H1.1 and H1.4 should be more effective in the binding RNA and forming structure of the ribosome. Likewise, binding of DNA can be mostly attributed to the subtype H1.2. Only two biological processes, i.e. biosynthetic (GO:0009058) and cellular nitrogen compound metabolic (GO:0034641), were indicated in the comparable proportions as related to the proteins partnering all of histone $\mathrm{H} 1$ subtypes. The interacting proteins of four histone H1 subtypes (H1.2, H1.3, H1.4 and H1.5) take part in the cellular protein modifications (GO:0036211), similarly to the proteins interacting with the subtype H1.1, H1.3, H1.4 and H1.5 that are involved in the signal transduction (GO:0007165). Both biological processes were detected as comparably abundant among histone $\mathrm{H} 1$ interacting proteins. A less frequently represented are biological processes characteristic of proteins interacting with a lower number of H1 subtypes. Besides translation (GO:0006412) assigned to the proteins interacting with subtype H1.1, H1.2 and H1.4, there are also processes characteristic of proteins partnering a single H1 subtype. Based on this, some of $\mathrm{H} 1$ subtypes might individually participate in the characteristic biological processes, i.e. H1.3 in the cell adhesion (GO:0007155) and a response to stress (GO:0006950) as well as H1.5 in the cell division (GO:0051301). A cell, nucleus and organelle was selected as main cellular component for all histone H1 interacting proteins. However, unlike the others H1 subtypes, subtype H1.4 possess partner proteins designated as a component of the ribosome. This may confirm a participation of this H1 subtype in the biological processes linked to the ribosome, i.e. ribosome biogenesis and translation, which were found as associated with its partnering proteins. A similar molecular functions and biological processes of proteins partnering the subtype H1.4 were recorded as enrichment by g:GOSt algorithm (Table 2). They include mainly molecular functions and biological processes coupled to the RNA binding and metabolism. The same analysis revealed a shared molecular functions of proteins interacting with histone H1.1 and H1.4, suggesting a functional closeness of both subtypes. Such a proximity may be also attributed to the subtype H1.1 and H1.2 due to an engagement of its partners in the chromosome organization (GO:0051276) and chromatin organization (GO:0006325). Interestingly, histone H1.2 as the only H1 subtype interact with proteins displaying enriched molecular function and biological process referring to the ubiquitin-dependent events, i.e. ubiquitin protein ligase binding (GO:0031625), ubiquitin-like protein ligase binding (GO:0044389) and protein modification by small protein conjugation (GO:0032446). The above findings that concern biological process and molecular function of proteins partnering histone H1 subtypes are closely associated with the pathways indicated in the Reactome database (Table 3).

\section{4 - Networks of histone H1 subtypes-partner proteins interactions}

Networks of histone H1 subtypes-partner proteins interactions were extracted from STRING database (v11.0) and introduced to the Cytoscape software (v3.8.0) for further analysis. A comparison of several topological parameters obtained by MCM function of Network Analyzer plugin indicate on an overall similarity of the networks $(F=0.613, p=0.654)$ (Table 4$)$. Although that some of analyzed topological parameters, such as characteristic path length, clustering coefficient, centralization and heterogeneity, possess the roughly similar numeric values, the differences are noticeable among the others. Because the networks sizes are defined ambiguously, e.g. small sized contain less than $10-20$ nodes ${ }^{29}$, small- and medium sized contain 50 -500 nodes ${ }^{30}$ and small to large sized contain 100 - 1000 nodes ${ }^{31}$, a concrete determination of H1 networks scales is rather intuitive because they differ three to four times on average. The subtype H1.1, H1.2 and H1.4 (126, 86 and 92 nodes) belong to greater networks compared to small scale networks of subtype H1.3 and H1.5 (30 and 47 nodes). Likewise, a network connectivity expressed by the average number of neighbors also differentiate the histone H1 subtype-interacting protein networks. The average connectivity of node, strongly related to the network resilience ${ }^{32}$, is about two and three times higher in the network of subtype H1.1 (13.872) and H1.4 (11.525) than in the network of subtype H1.5 (6.718) and H1.3 (3.6). These values characteristic of subtype H1.3 and H1.5 interaction network are also lower that of the subtype H1.2 (9.02). This indicate that the networks created by histone H1 subtypes differ in their compaction. Because highly connected nodes do not have a higher density ${ }^{33}$, the networks of subtype H1.1, H1.2 and H1.4 are more sparse than that of more dense of subtype H1.3 and H1.5. Another parameter, i.e. connected components indicating a connectivity of the network, is comparable for the networks of subtype H1.2, H1.3, H1.4 and H1.5 (in the range from 8 to 12), but its value is much lower (amounting 3) for the network created by 
histone H1.1. This pointing to a stronger connectivity of H1.1 subtype network compared to the networks of subtypes H1.2 - H1.5 ${ }^{34}$ and simultaneously reflecting their tendency to an increased fragmentation to the separated clusters. ${ }^{35}$ Most relevant neighborhood of histone H1 in the networks were identified by construction the active subnetworks using Diffusion algorithm of Cytoscape. In the vast majority, they contain proteins which GO terms that were predicted by CELLO2GO as associated with processes involved in the metabolism of DNA and regulation of its activity. It seems that in the DNA-dependent processes histone H1 subtypes displays a similar activity realized, however, by interactions with different proteins. The only one protein, i.e. DNA fragmentation factor subunit beta, turned out to be a component of subnetworks formed by subtypes H1.1, H1.3 and H1.5 (Table 5).

To find a highly connected nodes (hub proteins) with essential biological properties in the histone H1 subtypes-protein interaction networks, the MCM method of cytoHubba plugin was adopted. With the help of this tool, a top hub proteins of the networks were ranked (Table 6). An overview of the functions of selected proteins allow to designate the cell processes in which histone $\mathrm{H} 1$ subtypes might operate in common with their partnering proteins. Based on a similarity of molecular function and biological process of identified hubs, the histone H1 subtypes may be divided into three categories. First class includes subtypes H1.1 and H1.4 having five common proteins which, just like other top hubs, are involved in the RNA binding and biogenesis of ribosome. Second class contain subtypes H1.3 and H1.5 possessing three same proteins engaged in the control and regulation of cell cycle as well as in the cell division, similarly to the rest of the hubs. Third class encompass a single subtype H1.2 interacting with proteins responsible of protein ubiquitination and degradation. It should be noticed that among five histone H1 subtypes, the only subtype H1.3 was designated as a hub in the network. This suggest its critical role in the maintenance of the network structure. ${ }^{36}$ Thus, according to the centrality-lethality rule ${ }^{37}$, histone $\mathrm{H} 1.3$ may be recognized as functionally significant since its lack may disturb a pathway function. ${ }^{38}$ Moreover, a comparison of topological parameters values presented in the Table 6, indicated a disparity between the network constructed with subtype H1.1 and the networks created with subtype H1.3 and H1.5. A statistically significant difference was determined for a closeness centrality $(\mathrm{H} 1.1-\mathrm{H} 1.3 \mathrm{p}<0.05$ and H1.1 - H1.5 p $<0.01)$, clustering coefficient (H1.1 - H1.3 p $<0.05$ and H1.1 - H1.5 p < 0.05) and a neighborhood connectivity (H1.1 - H1.3 p < 0.001 and H1.1 - H1.5 $\mathrm{p}<0.001$ ). Such a distinctions between the networks hubs suggest functional dissimilarity between histone H1.1 and histone H1.3 and H1.5.

\section{5 - Structural and kinetic properties of complexes created by histone H1 subtypes and interacting protein}

In order to determine the types of complexes formed by histone H1 subtypes with interacting partners, the kinetic and structural parameters of binary interactions were evaluated. They include twelve proteins indicated in the APID database as displaying physical association with histone H1 subtypes, also annotated in the BioGRID and IntAct database (Figure 5). To discriminate a type of formed complexes, an occurrence of amino acid residues in the contact binding sites and its location in the secondary structure motifs were analyzed using BIPSPI and GOR method, respectively. Due to that the contact between proteins is reflected by increased proportion of differently structured amino acids ${ }^{39}$, the interface residues were grouped according to the biochemical and structural properties of side chain into hydrophobic (non-polar - Ala, Ile, Leu, Met, Phe, Pro, Trp, Val), polar (uncharged - Asn, Cys, Gly, Gln, Ser, Thr, Tyr) and charged (Asp, Arg, Glu, His, Lys). As it is seen in the Figure 5, in all interfaces of histone H1 subtypes prevailed are amino acid residues with charged (Arg, Glu and Lys) and polar (Asn, Gly, Ser and Thr) side chain. Only one amino acid containing a hydrophobic property is a residue of Ala that occur at a ratio amounting from 0.11 to 0.25 to the other residues. Thus, histone H1 subtypes favor the polar and charged interactions, mostly found in non-obligate ${ }^{40}$ and usually transient ${ }^{41}$ complexes. Because the transient complexes are weak associated and poorly optimized ${ }^{2}$, they might have at most a few hydrophobic residues which generally predominated in the obligate interaction. ${ }^{42}$ A specificity of $\mathrm{H} 1$ interactions is likely determined by large residues of Arg, Glu and Lys that make a side chain to side chain contact, compared to other residues, as Ser and Thr, which favor main chain to side chain contact. ${ }^{43} \mathrm{~A}$ complement of amino acid residues in the interfaces of H1-interacting proteins is essentially similar. For between protein-protein contact responsible are charged 
and polar residues, mainly Arg, Glu, Lys, Ser and Thr, which in a $75 \%$ of interactions are the sole residues of the interface. Occasionally, a residue of hydrophobic Leu and Met supplements an interacting surface. Such a transient type of histone H1-partner protein interaction inferred from a character of the interfaces residues was confirmed by determination of their positioning in the secondary structure elements. Whereas the regular secondary structures, i.e. $\alpha$-helix and $\beta$-sheets, are stabilized by hydrogen bonds between backbone atoms in not sequence specific mode, they are less important in formation the transient protein-protein interfaces. ${ }^{44}$ Compared to them, the non-regular elements, i.e. coils, loops and turns, are enriched in the transient interactions and specify this type of protein complex. ${ }^{41,42}$ However, both helical and non-regular secondary structures participating in various proportions in the transient interactions ${ }^{42}$ may form the mixed motifs characteristic of heterodimeric interfaces. ${ }^{45}$ The contact sites of histone H1 subtypes and their partnering proteins are completely devoid of $\beta$-sheets but participation of $\alpha$-helices is frequently observed. Although some of the binding sites are more abundant in the $\alpha$-helix fraction, the overall number of non-regular elements is higher. They occur in a $63.9 \%$ and $62.26 \%$ in the interfaces of histone H1 subtypes and their interacting proteins, respectively (Figure 6). In terms of the binding affinity, transient complexes can display a weak and strong interactions, commonly measured by difference in their equilibrium dissociation constant $\left(\mathrm{K}_{\mathrm{d}}\right) .{ }^{46}$ To define a strength of histone H1 subtypes-partner protein interactions, the binding affinity of emerging complexes was determined using the ISLAND predictor. The resulted values of $\mathrm{K}_{\mathrm{d}}$ for all complexes stay in the nanomolar range, from $10^{-8}$ to $10^{-7}$ (Table 7). According to the categorization of the binding affinities proposed by Veresov ${ }^{47}$, the complexes created by histone H1 subtypes should be classified as medium strong transient, for which $\mathrm{K}_{\mathrm{d}}$ takes a value between $10^{-8}$ and $10^{-6}$. Thus, histone H1 subtypes together with partner proteins fall in the transient interactions by forming a medium unstable complexes. They are capable of temporal association and dissociation in dependence on the effects caused by specific stabilizers and triggers, respectively, that determine a characteristic conformational state. ${ }^{48}$

\section{6 - Intrinsic disorder of histone H1 subtypes-interacting protein complexes}

Because transient interactions were frequently presented as coupled to the protein disorder ${ }^{48,49,50}$, it seems advisable to check in which extend such a phenomena influence on histone H1 subtypes interactions. In order to found the disordered regions in the interfaces of physically associated proteins, their sequences were analyzed with the DisEMBL predictor. For all of the contact regions in the histone H1 subtypes, a characteristic is an occurrence of disordered residues, in the amount from $50 \%$ to $80 \%$. On the other hand, among the histone $\mathrm{H} 1$ interacting proteins are six having fully ordered interfaces and six ones possessing from $83 \%$ to $100 \%$ of the disordered residues (Figure 5). While histone H1 subtypes were already recognized as abundantly disordered proteins displaying the representative features of sequence and structure attributable to the intrinsic structural disorder ${ }^{51}$, a way of their interactions and a kind of formed complexes remained unknown. The current analysis allowed to show that histone H1 subtypes may create two types of complexes via disordered motifs. According to the categories of complexes introduced by Mészáros et al. ${ }^{52}$, the first encompass interaction between disordered $\mathrm{H} 1$ and ordered partner through the coupled folding and binding and the second include interaction between disordered $\mathrm{H} 1$ and disordered partner through the mutual synergistic folding. If so, histone $\mathrm{H} 1$ have potential for creation a transient (weaker) interaction with ordered partner due to the decreasing binding strength evoked by loss of conformational entropy ${ }^{53}$ as well as to form more stable complexes with disordered partner due to a difference in the compositions and variation of the backbone sequence. ${ }^{54} \mathrm{~A}$ participation of histone $\mathrm{H} 1$ in the weaker interactions results from its intrinsic disorder reaching above $90 \%$ of the sequence, while a share in the stronger ones provides a presence of MoRFs and APBRs motifs stabilizing the interactions. ${ }^{51}$ Thus, it seems that a specificity of interaction is determined by an individual sequence and/or structure properties of the binding partner. By generalizing, this finding confirm that intrinsic disorder is an inherent feature of histone $\mathrm{H} 1$ subtypes, essential for recognition and binding of an interacting partner. A disorder-dependent formation of various complexes seems to be crucial for multifaceted functioning of histone $\mathrm{H} 1$ subtypes.

\section{4 - Histone H1 subtypes characteristics inferred from protein-protein interactions}

The notion that histone $\mathrm{H} 1$ act in the complexes with proteins has been earlier confirmed by in vivo and in 
vitro experiments on over a dozen examples, including mainly interactions involved in the dynamic changes of chromatin states. ${ }^{55}$ However, the subsequent investigation performed on the proteome-scale revealed almost two hundred of proteins which may bind to the histone H1. Analyses conducted by Kalashnikova et al. ${ }^{56}$ and Szerlong et al. ${ }^{57}$ indicate that histone $\mathrm{H} 1$ is essential for organize the nucleolus protein network needed, among others, for RNA splicing and ribosome biogenesis. These are the first studies documenting such a wide range of possible histone $\mathrm{H} 1$ interactions relating, however, only to two histone $\mathrm{H} 1$ variants purified from cultured cells. Considering that the human H1 histones display a highest degree of heterogeneity manifested by a presence of 11 variants $^{58}$, a complete picture of $\mathrm{H} 1$ interactions remained unknown. This work helps in determination of a scale in which histones $\mathrm{H} 1$ might be active, simultaneously indicating on the mechanisms of their operation. Because the computational analysis include effective methods for prediction a biological significance of protein-protein interactions ${ }^{59}$, the sequence-based and structure-based approaches were adopted for study of the interactions between histone H1 subtypes and partnering proteins.

A characteristic of histone $\mathrm{H} 1$ subtypes interacting proteins location presented in this work indicate that they have no preferences for an occurrence in the nucleolus, in contrast to the subtype H1.0 for which 175 nucleolar interacting partners were identified. ${ }^{56}$ The partners of subtypes H1.1 - H1.5 are mainly nuclear and cytosolic proteins but in the lower proportions remaining also in the membrane and mitochondrion. Thus, it can be predicted that the functions of subtypes H1.1 - H1.5 are predominantly realized in these cellular compartments. The obtained data regarding functions confirm a shared way of histone H1 subtypes action but simultaneously indicate on new aspects of their individualization. A clarification of distinction between commonality and specificity can be observed in the juxtaposition of $\mathrm{H} 1$ interacting proteins features presented in the Figure 6. Based on the selected criteria including molecular function, biological processes and the networks properties, histone $\mathrm{H} 1$ subtypes can be categorized according to tendency of similar and/or dissimilar activity. A frequently occurring are the common features of subtypes H1.1 and H1.4 (first category) and subtypes H1.3 and H1.5 (second category), sometimes also characteristic of subtype H1.2 (third category). Due to this, an activity of the said pairs of subtypes is peculiarly limited to the selected processes but, on the other hand, it can be realized in a similar way. In contrast, the subtype H1.2 repeatedly appearing together with a pair of H1.1, H1.4 and/or H1.3, H1.5 should be treated as more ubiquitous and, thus, more capable of engagement in a broader range of the activity. This stay in agreement with suggestion that histone $\mathrm{H} 1$ subtypes may share functionally redundant roles in some of the biological processes but also act specifically in the other ones. ${ }^{25}$ However, such a grouped activities of H1 subtypes determined in this work deviates from those which are characteristic of the DNA-associated processes in the chromatin. For example, the strength of binding to the DNA is high in the case of subtype H1.3 and H1.4 and decreases to intermediate for subtype H1.2 and H1.5 and to the low for subtype H1.1. ${ }^{60}$ Likewise, the strong chromatin binging characterize subtypes H1.4 and H1.5, in contrast to intermediate binding by subtype H1.3 and weak binding by subtype H1.1. ${ }^{61}$ It seems that histone H1 subtypes may manifest various types of activities in distinct cellular processes, in dependence of the contacts with the DNA and proteins. The identified disparities between histone $\mathrm{H} 1$ subtypes are also reflected by their presence in the various networks coordinating a run of critical cell processes, i.e. ribosome biogenesis (subtype H1.1 and H1.4), cell cycle (subtype H1.3 and H1.5) and protein degradation (subtype H1.2). However, the most important in this field is a function of histone H1.3. As a hub protein, it play a central role in the molecular organization of the network and is useful to highlight nodes with special biological functions. ${ }^{29}$ It seem that the histone H1.3 may be considered as date (dynamic) hub. Due to a high content of intrinsic structural disorder ${ }^{51}$ attributable mainly to the dynamic hubs ${ }^{62}$, it can bind its interaction partners in the times and/or location-specific mode. ${ }^{38}$

A type of histone $\mathrm{H} 1$ subtypes interactions, transient in term of the stability and medium-strong in relation to the strength of binding, is frequent in the biochemical pathways and regulatory networks and, therefore, it might be considered as important in a range of cellular functions. ${ }^{2}$ Such a dynamic and reversible interactions are typically accompanying with conformational changes of proteins ${ }^{63}$, so they are usually control by their intrinsically disordered sequence stretches. ${ }^{48}$ This also applies to the histones H1 which may bind both disordered and ordered proteins through a disordered interaction surface. In this context, intrinsic disorder might be considered as prerequisite to creation and maintenance of histone H1 interaction networks. 
However, the mode of binding appropriate for histones H1 can be also influenced by other stimuli, mainly by post-translational modifications ${ }^{52}$ attributable to the intrinsic disordered state. ${ }^{64}$ Because the modified residues, i.e. acetylated, methylated and phosphorylated, were detected in the histones H1 disordered terminal domains ${ }^{65}$, it can be assumed that histone $\mathrm{H} 1$ subtypes interactions might be influenced by a cooperative regulation of acetylation and methylation. ${ }^{52}$

\section{5 - CONCLUSIONS}

The properties of histone H1 subtypes presented in this study allow to define them as multifunctional proteins that serve as an elements coordinating a plethora roles of the cell. Through the interactions with diversely active proteins, histone H1 subtypes build the complex networks orchestrated crucial cellular processes including cell cycle, ribosome biogenesis, translation and degradation of proteins. The individual and/or common (partially redundant) activity of histone H1 subtypes is realized by forming transient protein complexes through the disordered regions. Ultimately, the gathered evidences of multifaceted histone H1 subtypes functioning realized together with partnering proteins confirms a legitimacy of introducing an extended histone $\mathrm{H} 1$ paradigm relating to its operation through the protein-protein interactions. ${ }^{8}$

ORCID Andrzej Kowalski https:/ /orcid.org/0000-0001-8856-5863

\section{REFERENCES}

1. De Las Rivas J, Fontanillo C. Protein-protein interaction networks: unraveling the wiring of molecular machines within the cell. Brief Funct Genomics . 2012;11:489-96.

2. Ozbabacan SEA, Engin HB, Gursoy A, Keskin O. Transient protein-protein interactions. Protein Eng Des Sel . 2011;24:635-648.

3. Braun P, Gingras A-C. History of protein-protein interaction: from egg-white to complex networks. Proteomics . 2012;12:1478-1498.

4. Torrente MP, Zee BM, Young NL, Baliban RC, LeRoy G, Floudas CA. Proteomic Interrogation of Human Chromatin. PLoS ONE . 2011;6:e24747.

5. Zhou B-R, Bai Y. Chromatin structures condensed by linker histones.Essays Biochem . 2019; 63:75-87.

6. Kowalski A, Pałyga J. Modulation of chromatin function through linker histone H1 variants. Biol Cell . 2016;108:1-18.

7. Fyodorov DV, Zhou B-R, Skoultchi AI, Bai Y. Emerging roles of linker histones in regulating chromatin structure and function. Nature Rev Mol Cell Biol . 2017;19:192-206.

8. Kalashnikova AA, Rogge RA, Hansen JC. Linker histone H1 and protein-protein interactions. Biochim Biophys Acta . 2016;1859:455-461.

9. Alonso-López D, Campos-Laborie FJ, Gutiérrez MA, Lambourne L, Calderwood MA, Vidal M, De Las Rivas J. APID database: redefining protein-protein interaction experimental evidences and binary interactomes. Database . 2019;1-8.

10. Oughtred R, Stark C, Breitkreutz B-J, Rust J, Boucher L, Chang C, Kolas N, O'Donnell L, Leung G, McAdam R. The BioGRID interaction database: 2019 update. Nucleic Acids Res . 2019;47:529-541.

11. Orchard S, Ammari M, Aranda B, Breuza L, Briganti L, Broackes-Carter F, Campbell NH, Chavali G, Chen C, del-Toro N. The MIntAct project-IntAct as a common curation platform for 11 molecular interaction databases. Nucleic Acids Res. 2014;42:358-363.

12. The UniProt Consortium. UniProt: the universal protein knowledgebase. Nucleic Acids Res . 2017;45:158169. 
13. Veres DV, Gyurkó DM, Thaler B, Szalay KZ, Fazekas D, Korcsmáros T, Csermely P. ComPPI: a cellular compartment-specific database for protein-protein interaction network analysis. Nucleic Acids Res . $2015 ; 43: 485-493$.

14. Sahraeian SME, Luo KR, Brenner SE. SIFTER search: a web server for accurate phylogeny-based protein function prediction. Nucleic Acids Res . 2015;43: 141-147.

15. Yu C-S, Cheng C-W, Su W-C, Chang K-C, Huang S-W, Hwang J-K, Lu C-H. CELLO2GO: A Web Server for Protein subCELlular LOcalization Prediction with Functional Gene Ontology Annotation. PLoS ONE . 2014;9:e99368.

16. Raudvere U, Kolberg L, Kuzmin I, Arak T, Adler P, Peterson H, Vilo J. g:Profiler: a web server for functional enrichment analysis and conversions of gene lists (2019 update) Nucleic Acids Res . 2019;47:191198.

17. Szklarczyk D, Gable AL, Lyon D, Junge A, Wyder S, Huerta-Cepas J, Simonovic M, Doncheva NT, Morris JH, Bork P, Jensen LJ, von Mering C. STRING v11: protein-protein association networks with increased coverage, supporting functional discovery in genome-wide experimental datasets.Nucleic Acids Res . 2019;47:607-613.

18. Asssenov Y, Ramirez F, Schelhorn SE, Langauer T, Albrecht M. Computing topological parameters of biological network. Bioinformatics . 2008;242:282-284.

19. Chin C-H, Chen S-H, Wu H-H, Ho C-W, Ko M-T, Lin C-Y. cytoHubba: identifying hub objects and sub-networks from complex interactome.BMC Syst Biol . 2014;8 (Suppl 4):11.

20. Carlin DE, Demchak B, Pratt D, Sage E, Ideker T. Network propagation in the cytoscape cyberinfrastructure. PLoS Comput Biol . 2017;13(10):e1005598.

21. Sanchez-Garcia R, Sorzano COS, Carazo JM, Segura J. BIPSPI: a method for the prediction of partnerspecific protein-protein interfaces.Bioinformatics . 2019;35:470-477.

22. Linding L, Jensen LJ, Diella F, Bork P, Gibson TJ, Russell RB. Protein disorder prediction: implications for structural proteomics. Structure . 2003;11:1453-1459.

23. Taner Z, Sen R, Jernigan L, Garnier J, Kloczkowski A. GOR V server for protein secondary structure prediction. Bioinformatics . 2005;21:2787-2788.

24. Abbasi WA, Hassan FU, Yaseen A, Minhas FUAA. ISLAND: In-silico prediction of proteins binding affinity using sequence descriptors. arXiv preprint . 2017;arXiv:1711.10540.

25. Millãn-Ariño L, Izquierdo-Bouldstridge A, Jordan A. Specificities and genomic distribution of somatic mammalian histone H1 subtypes. Biochim Biophys Acta . 2016;1859:510-519.

26. Izzo A, Kamieniarz-Gdula K, Ramirez F, Noureen N, Kind J, Manke T, van Steensel B, Schneider R. The genomic landscape of the somatic linker histone subtypes H1.1 to H1.5 in human cells. Cell Rep . 2013;3:2142-2154.

27. Millán-Ariño L, Islam ABMMK, Izquierdo-Bouldstridge A, Mayor R, Terme J-M, Luque N, Sancho M, López-Bigas N, Jordan A. Mapping of six somatic linker histone H1 variants in human breast cancer cells uncovers specific features of H1.2. Nucleic Acids Res . 2014;42:4474-4493.

28. Kim J, Kim K, Punj V, Liang G, Ulmer TS, Lu W, An W. Linker histone H1.2 establishes chromatin compaction and gene silencing through recognition of H3K27me3. Sci Rep . 2015; 5:16714.

29. Agapito G, Guzzi PH, Cannataro M. Visualization of protein interaction networks: problems and solutions. BMC Bioinformatics . 2013;14:S1.

30. Merico D, Gfeller D, Bader GD. How to visually interpret biological data using networks. Nat Biotechnol . 2009;27:921-924. 
31. Xia J, Benner MJ, Hancock REW. NetworkAnalyst - integrative approaches for protein-protein interaction network analysis and visual exploration. Nucleic Acids Res . 2014;42:167-174.

32. Celestini A, Cianfriglia M, Mastrostefano E, Palma A, Castiglione F, Tieri P. Critical nodes reveal peculiar features of human essential genes and protein interactome. IEEE International Conference on Bioinformatics and Biomedicine (BIBM), San Diego, CA, USA, 2019;2121-2128.

33. Almaas E. Biological impact and context of network theory. J Exp Biol . 2007;210:1548-1558.

34. Hakes L, Pinney J, Robertson D, Lovell SC. Protein-protein interaction networks and biology - what's the connection?. Nat Biotechnol . 2008;26:69-72.

35. Friedel CC, Zimmer R. Influence of degree correlations on network structure and stability in proteinprotein interaction networks. BMC Bioinformatics . 2007;8:297.

36. He X, Zhang J. Why do hubs tend to be essential in protein networks ? PLoS Genet . 2006;2(6):e88.

37. Jeong H, Mason SP, Barabasi A-L, Oltvai ZN. Lethality and centrality in protein network. Nature . 2001;411:41-42.

38. Han J-DJ, Bertin N, Hao T, Goldberg DS, Berriz GF, Zhang LV, Dupuy D, Walhout AJM, Cusick ME, Roth FP, Vidal M. Evidence of dynamically organized modularity in the yeast protein interaction network. Nature . 2004;430:88-93.

39. Veselovsky AV, Ivanov YD, Ivanov AS, Archakov AI, Lewi P, Janssen P. Protein-protein interactions: mechanisms and modification by drugs.J Mol Recognit . 2002;15:405-422.

40. Park SH, Reyes JH, Gilbert DR, Kim JW, Kim S. Prediction of protein-protein interaction types using association rule based classification. BMC Bioinformatics . 2009;10:36.

41. La D, Kong M, Hoffman W, Choi YI, Kihara D. Predicting permanent and transient protein-protein interfaces. Proteins . 2013;81:805-815.

42. De S, Krishnadev O, Srinivasan N, Rekha N. Interaction preferences across protein-protein interfaces of obligatory and non-obligatory components are different. BMC Struct Biol . 2005;5:15.

43. Talavera D, Robertson DL, Lovell SC. Characterization of protein-protein interaction interfaces from a single species. PLoS One . 2011;6(6):e21053.

44. Ansari S, Helms V. Statistical analysis of predominantly transient protein-protein interfaces. Proteins . 2005;61:344-355.

45. Guharoy M, Chakrabarti P. Secondary structure based analysis and classification of biological interfaces: identification of binding motifs in protein-protein interactions. Bioinformatics . 2007;23:1909-1918.

46. Nooren IMA, Thornton JM. Structural characterization and functional significance of transient proteinprotein interactions. J Mol Biol . 2003;325:991-1018.

47. Veresov VG. Predicting protein-protein binding affinity by in silico docking. JSM Chem . 2016;4:1019.

48. Perkins JR, Diboun I, Dessaily BH, Lees JG, Orengo C. Transient protein-protein interactions: structural, functional and network properties. Structure . 2010;18:1233-1243.

49. Pancsa R, Fuxreiter M. Interactions via intrinsically disordered regions: what kind of motifs ? IUBMB Life . 2012;64:5130-520.

50. Singh GP, Ganapathi M, Dash D. Role of intrinsic disorder in transient interactions of hub proteins. Proteins . 2007;66:761-765.

51. Kowalski A. Abundance of intrinsic structural disorder in the histone H1 subtypes. Comput Biol Chem . 2015;59:16-27. 
52. Mészáros B, Dobson L, Fichó E, Tusnády GE, Dosztányi Z, Simon I. Sequential, structural and functional properties of protein complexes are defined by how folding and binding intertwine. J Mol Biol . 2019;431:44084428.

53. Chu X, Wang J. Specificity and affinity quantification of flexible recognition from underlying energy landscape topography. PLoS Comput Biol . 2014;10,:e1003782.

54. Mentes A, Magyar C, Fichó E, Simon I. Analysis of heterodimeric "Mutual Synergistic Folding" - complexes. Int J Mol Sci . 2019;16:5136.

55. McBryant S. Lu X, Hansen JC. Multifunctionality of the linker histones: an emerging role for proteinprotein interactions. Cell Res . 2010;20:519-528.

56. Kalashnikova AA, Winkler DD, McBryant SJ, Henderson RK, Herman JA, DeLuca JG, Luger K, Prenni JE, Hansen JC. Linker histone H1.0 interacts with an extensive network of proteins found in the nucleolus.Nucleic Acids Res . 2013;41:4026-4035.

57. Szerlong HJ, Herman JA, Krause CM, DeLuca JG, Skoultchi A, Winger QA, Prenni JE, Hansen JC. Proteomic characterization of the nucleolar linker histone H1 interaction network. J Mol Biol . 2015;427:20562071.

58. Talbert PB, Ahmad K, Almouzni G, Ausio J, Berger F, Bhalla PL, Bonner WM, Cande WZ, Chadwick B, Chan SWL, Cross GAM, Cui L, Dimitrov SI, Doenceke D, Eirin-Lopez JM, Gorovsky MA, Hake SB, Hamkalo BA, Holec S, Jacobsen SE, Kamieniarz K, Kchohbin S, Ladurner AG, Landsman D, Latham JA, Loppin B, Malik HS, Marzluff WF, Pehrson JR, Postberg J, Schneider R, Singh M, Smith MM, Thompson E, Torres-Padilla M-E, Tremethick DJ, Turner BM, Waterborg JH, Wollmann H, Yelagandula R, Zhu B, Henikoff S. A unified phylogeny-based nomenclature for histone variants. Epigenetics \& Chromatin . 2012;5:7.

59. Folador EL, Fernandez de Oliveira Junior A, Tiwari S, Jamal SB, Ferreira FS, Barh D, Ghosh P, Ilva A, Azevedo V. In silicoprotein-protein interactions: avoiding data and methods biases over sensitivity and specificity. Curr Protein Pep Sc . 2015;16:689-700.

60. Orrego M, Ponte I, Roque A, Buschati N, Mora X, Suau P. Differential affinity of mammalian histone H1 somatic subtypes for DNA and chromatin. BMC Biol . 2007;5:22.

61. Clausell J, Happel N, Hale TK, Doenecke D, Beato M. Histone H1 subtypes differently modulate chromatin condensation without preventing ATP-dependent remodeling by SWI/SNF or NURF. PLoS One . 2009;4(10):e0007243.

62. Patil A, Kinoshita K, Nalamura H. Hub promiscuity in protein-protein interaction networks. Int J Mol Sci . 2010;11:1930-1943.

63. Janin J, Bahadur RP, Chakrabarti P. Protein-protein interaction and quaternary structure. Q Rev Biophys . 2008;41:133-180.

64. Darling AL, Uversky VN. Intrinsic disorder and posttranslational modifications: the darker side of the biological dark matter.Front Genet . 2018;9:158.

65. Rogue A, Ponte I, Suau P. Post-translational modifications of the intrinsically disordered terminal domains of histone H1: effects on secondary structure and chromatin dynamics. Chromosoma. 2016;126:83-91.

Caption for figures

FIGURE 1 Schematic framework of data searching and processing

FIGURE 2 Quantitative assessment of proteins interacting with histone H1 subtypes. On A, darkened bars shows a total number of proteins and brightened bars shows the number of proteins individual for a given subtype. On B, pairwise grouping of histone $\mathrm{H} 1$ subtypes based on the amount of common interacting proteins 
FIGURE 3 Localization of histone H1 subtypes interacting proteins determined with the ComPPI database. Abundance of proteins was expressed as a ratio of their amount in a given cell compartment to the whole number of proteins interacting with a given histone $\mathrm{H} 1$ subtype

FIGURE 4 Functions of histone H1 subtypes interacting proteins derived from the SIFTER database. Abundance of proteins assigned to functional category was expressed as a ratio of their amount to the whole number of proteins interacting with a given histone $\mathrm{H} 1$ subtype

FIGURE 5 Interface residues of histone H1 subtypes and their interacting proteins predicted by BIPSPI method. In parentheses, an amino acid side chain propensity (subscript: $\mathrm{c}$ - charged, $\mathrm{p}$ - polar and $\mathrm{h}-$ hydrophobic) and its location in secondary structure (superscript: $\mathrm{H}$ - helix, NR - Non-Regular) is depicted. Underlined residues were predicted by DisEMBL tool as disordered

FIGURE 6 Features of histone H1 interacting proteins indicating on a shared and distinct characteristic of histone H1 subtypes. Similarities and distinctions between molecular function and biological process were juxtaposed based on proportions of proteins frequently associated with a given feature and having comparable networks properties (darkened circles) versus the proteins rarely and/or completely unrelated to a given feature and having unlike networks properties (lightened circles), details in the text

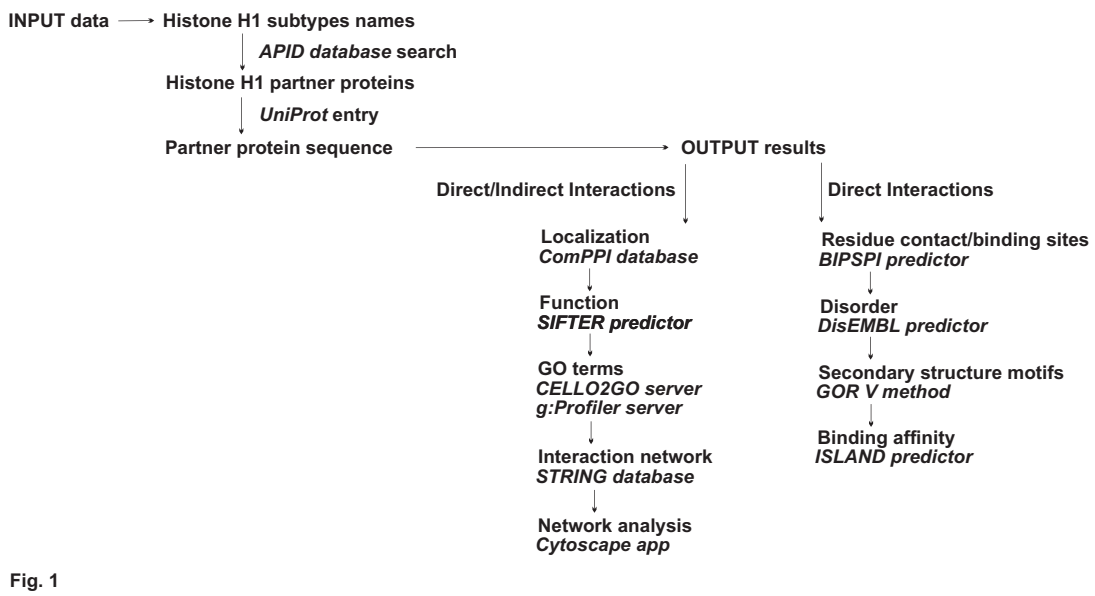


A

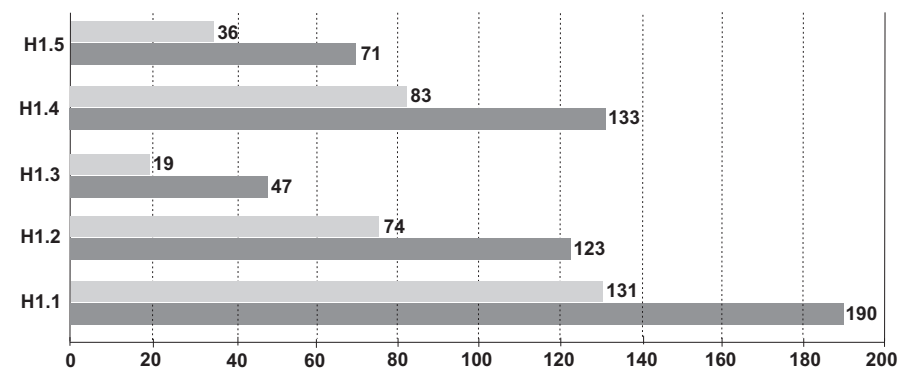

B

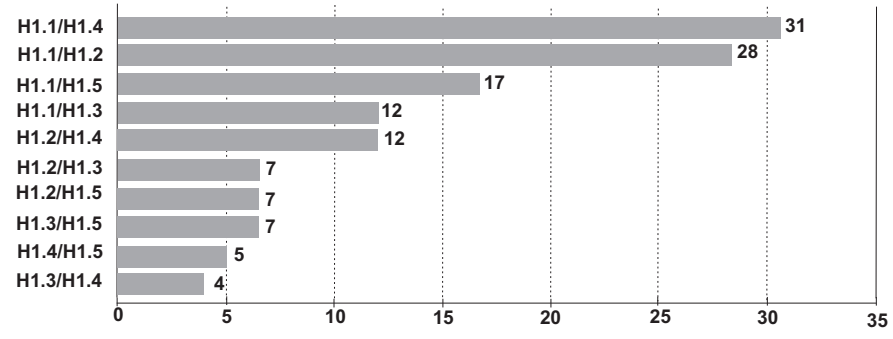

Fig. 2 


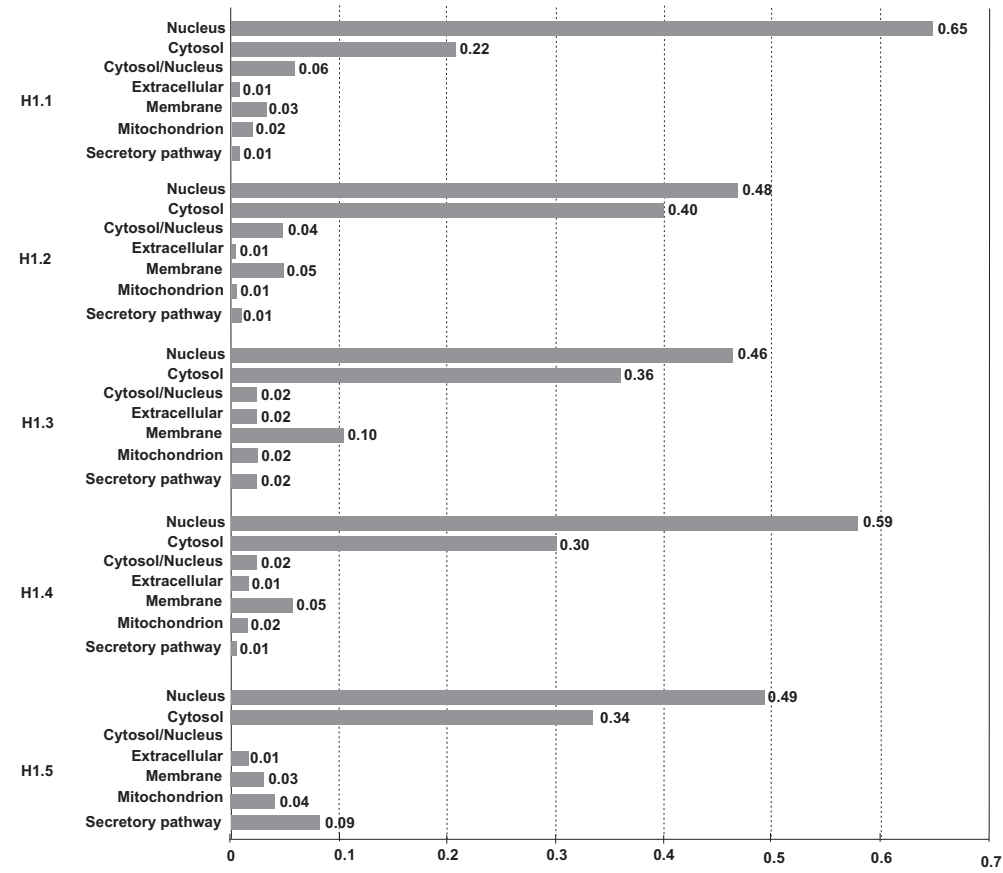

Fig. 3 
H1.1

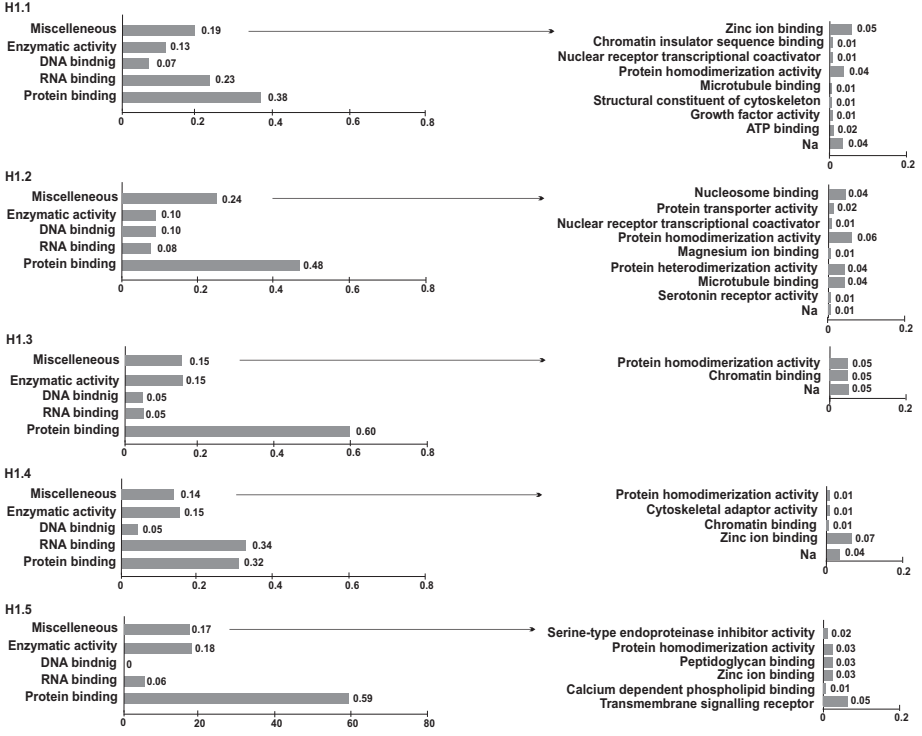

Fig. 4

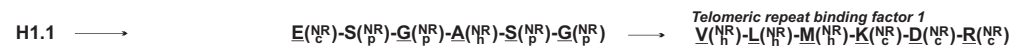

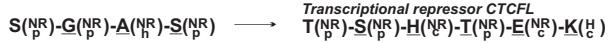

Fig. 5
} 


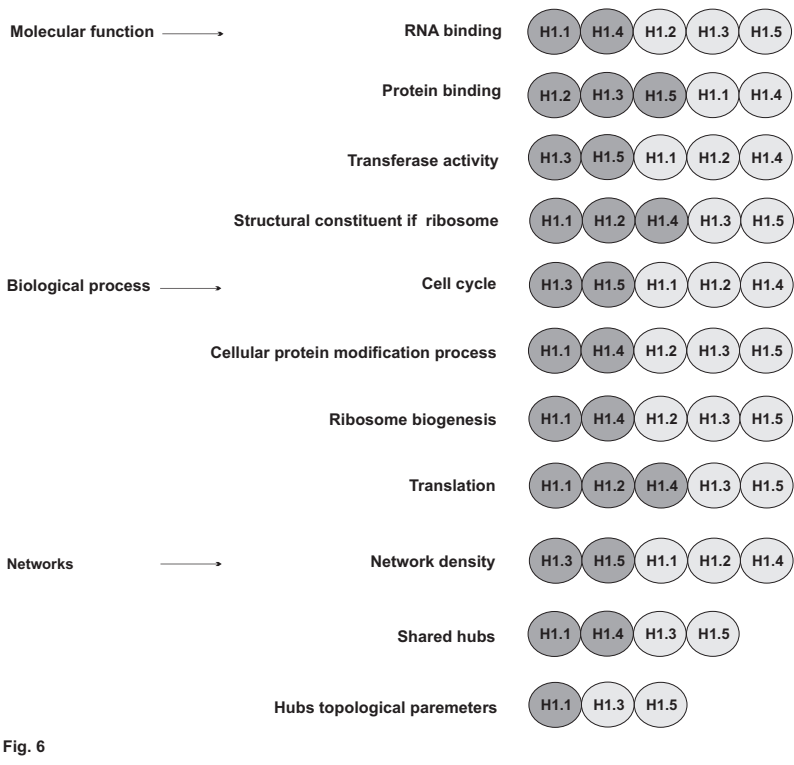

\section{Hosted file}

Table 1.pdf available at https://authorea.com/users/361445/articles/482863-a-survey-ofhuman-histone-h1-subtypes-interaction-networks-implications-for-histones-h1-functioning

\section{Hosted file}

Table 2.pdf available at https://authorea.com/users/361445/articles/482863-a-survey-ofhuman-histone-h1-subtypes-interaction-networks-implications-for-histones-h1-functioning

\section{Hosted file}

Table 3.pdf available at https://authorea.com/users/361445/articles/482863-a-survey-ofhuman-histone-h1-subtypes-interaction-networks-implications-for-histones-h1-functioning

\section{Hosted file}

Table 4.pdf available at https://authorea.com/users/361445/articles/482863-a-survey-ofhuman-histone-h1-subtypes-interaction-networks-implications-for-histones-h1-functioning

\section{Hosted file}

Table 5.pdf available at https://authorea.com/users/361445/articles/482863-a-survey-ofhuman-histone-h1-subtypes-interaction-networks-implications-for-histones-h1-functioning

\section{Hosted file}

Table 6.pdf available at https://authorea.com/users/361445/articles/482863-a-survey-ofhuman-histone-h1-subtypes-interaction-networks-implications-for-histones-h1-functioning

\section{Hosted file}

Table 7.pdf available at https://authorea.com/users/361445/articles/482863-a-survey-ofhuman-histone-h1-subtypes-interaction-networks-implications-for-histones-h1-functioning 